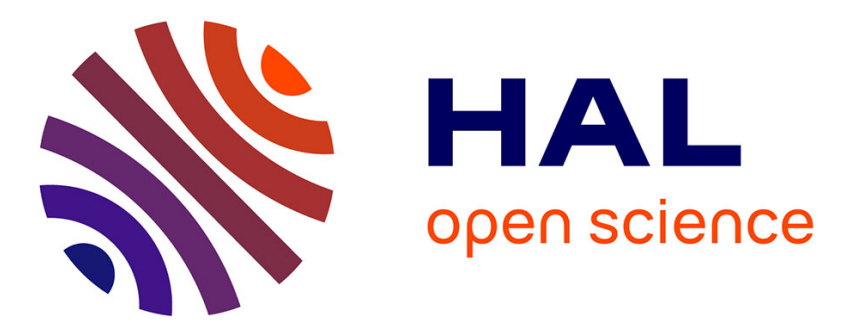

\title{
Auto-sondages intermittents et rétentions urinaires des syndromes parkinsoniens
}

Etienne Savard, Arnaud Declemy, Valentin Coindreau, Florence Babany, Camille Chesnel, Audrey Charlanes, Frédérique Le Breton, Gérard Amarenco

\section{- To cite this version:}

Etienne Savard, Arnaud Declemy, Valentin Coindreau, Florence Babany, Camille Chesnel, et al.. Autosondages intermittents et rétentions urinaires des syndromes parkinsoniens. Progrès en Urologie, 2018, 28 (17), pp.987-992. 10.1016/j.purol.2017.11.004 . hal-02309525

\section{HAL Id: hal-02309525 \\ https://hal.sorbonne-universite.fr/hal-02309525}

Submitted on 9 Oct 2019

HAL is a multi-disciplinary open access archive for the deposit and dissemination of scientific research documents, whether they are published or not. The documents may come from teaching and research institutions in France or abroad, or from public or private research centers.
L'archive ouverte pluridisciplinaire HAL, est destinée au dépôt et à la diffusion de documents scientifiques de niveau recherche, publiés ou non, émanant des établissements d'enseignement et de recherche français ou étrangers, des laboratoires publics ou privés. 
urologie

Elsevier Editorial System(tm) for Progrès en

Manuscript Draft

Manuscript Number:

Title: Self-intermittent-catheterization and urinary retention in Parkinson diseases Autosondages intermittents et rétentions urinaires des syndromes parkinsoniens.

Article Type: Article original

Section/Category: Pelvi-périnéologie

Keywords: Mots clés : "syndromes parkinsoniens », « autosondage », "rétention urinaire »

Key words : Parkinson disease, urinary retention, self-intermittentcatheterization

Corresponding Author: Dr. Etienne SAVARD,

Corresponding Author's Institution: APHP

First Author: Etienne SAVARD

Order of Authors: Etienne SAVARD; Arnaud Declemy; Florence Babany; Valentin Coindreau; Audrey Charlanes, MD; Camille Chesnel, MD; Frédérique Le Breton; Gérard Amarenco, PhD

Abstract: Abstract

Introduction : Lower urinary tract symptoms are common in Parkinson diseases, especially chronic urinary retention. In case of significant and symptomatic post void residual, a specific treatment is necessary in order to empty the bladder and nowadays the gold standard of a such neurogenic bladder is based on self-intermittent-catheterizations if possible at all. We carried out a retrospective study about feasibility and outcomes of self-intermittent-catheterizations in this population. Methods : Retrospective study with qualitative and quantitative, clinical and instrumental (urodynamic) assessment, of lower urinary tract symptoms as urinary retention in extrapyramidal syndromes.

Results : 42 patients with parkinsonian syndrome performing selfintermittent-catheterization were assessed. Twenty-one had idiopathic Parkinson's Disease, 17 Multiple System Atrophy, 1 vascular Parkinson, 1 iatrogenic Parkinson and 2 not yet determined parkinsonian syndromes. All the patients had urinary retention characterized by a post-voiding residual volume more than $150 \mathrm{ml}$. All the patients were symptomatic and reported voiding dysfunction (30/42), overactive bladder syndrome $(20 / 42)$, stress urinary incontinence (6/42). The cystometry showed detrusor overactivity (15/42), bladder-sphincter dyssynergia (15/42), detrusor underactivity (14/42), sphincter deficiency (4/42) or bladder compliance alteration (3/42). Each patient have learned and well controlled self-intermittent-catheterization technique. Fourteen (33\%) stopped self-intermittent-catheterization prematurely in the following months because of functional impact of neurological worsening. 
Conclusion : Even if one third of the patients had stopped self-

intermittent-catheterization because of neurological deterioration, this technique remains the gold standard for the treatment of urinary chronic retention in parkinsonian patients.

Key words : Parkinson disease, urinary retention, self-intermittentcatheterization

\section{Résumé}

Introduction : Les troubles vésico-sphinctériens sont fréquents dans les syndromes parkinsoniens, notamment la rétention urinaire chronique. En cas de résidu significatif et symptomatique se pose le problème de son traitement qui se résume le plus souvent à la faisabilité ou non de la pratique des auto-sondages intermittents. Nous avons conduit une étude rétrospective sur la faisabilité et les résultats des auto-sondages dans cette population.

Méthodes : Étude rétrospective avec analyse qualitative et quantitative, clinique et instrumentale (urodynamique), des troubles vésico-

sphinctériens à type de rétention au cours des syndromes extrapyramidaux. Résultats : 42 patients avec syndrome parkinsonien et réalisant des autosondages intermittents ont été examinés. Vingt-et-un étaient atteints d'une maladie de Parkinson idiopathique, 17 d'une atrophie multisystématisée, 1 d'un syndrome parkinsonien d'origine vasculaire, 1 d'un syndrome parkinsonien d'origine iatrogène et 2 d'un syndrome extrapyramidal non étiqueté. Tous avaient une rétention d'urine définie par un résidu post-mictionnel supérieur ou égal à cent cinquante millilitres. Tous étaient symptomatiques et les symptômes rapportés étaient une dysurie (30/42), une hyperactivité vésicale (20/42) et une incontinence urinaire d'effort (6/42). La cystomanométrie mettait en évidence une hyperactivité détrusorienne (15/42), une dyssynergie vésicosphinctérienne (15/42), une hypo-contractilité détrusorienne (14/42), une incompétence sphinctérienne (4/42) ou un défaut de compliance (3/42). Tous les patients maitrisaient la technique des auto-sondages intermittents. Quatorze (33\%) ont arrêté l'auto-sondage précocement dans les mois qui suivaient l'apprentissage en raison du retentissement fonctionnel de la dégradation neurologique.

Conclusion : Même si un tiers des patients abandonnent la réalisation des auto-sondages en raison de l'aggravation de la maladie neurologique, ces derniers restent la méthode de choix du traitement symptomatique de la rétention chronique du patient parkinsonien.

Mots clés : « syndromes parkinsoniens », « auto-sondage », « rétention urinaire »

Suggested Reviewers: 


\title{
Auto-sondages intermittents et rétentions urinaires des syndromes parkinsoniens
}

Self-intermittent-catheterization and urinary retentions in parkinsonian syndromes

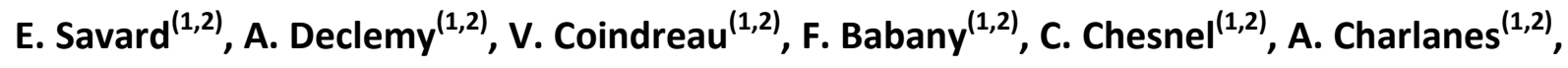 \\ F. Le Breton ${ }^{(1,2)}$, G. Amarenco ${ }^{(1,2)}$
}

1. GREEN GRC-01 UPMC (Groupe de Recherche Clinique en Neuro-Urologie), Sorbonne Universités, Paris, France

2. Service de Neuro-Urologie, Hôpital Tenon, APHP, Paris, France

Auteur correspondant :

Etienne SAVARD

Service de Neuro-Urologie

Hôpital Tenon, 4 rue de la Chine, 75020 Paris France

etienne.savard@aphp.fr 


\section{Auto-sondages intermittents et rétentions urinaires des syndromes parkinsoniens}

\section{Résumé}

Introduction: Les troubles vésico-sphinctériens sont fréquents dans les syndromes parkinsoniens, notamment la rétention urinaire chronique. En cas de résidu significatif et symptomatique se pose le problème de son traitement qui se résume le plus souvent à la faisabilité ou non de la pratique des auto-sondages intermittents. Nous avons conduit une étude rétrospective sur la faisabilité et les résultats des auto-sondages dans cette population.

Méthodes: Étude rétrospective avec analyse qualitative et quantitative, clinique et instrumentale (urodynamique), des troubles vésico-sphinctériens à type de rétention au cours des syndromes extrapyramidaux.

Résultats: 42 patients avec syndrome parkinsonien et réalisant des auto-sondages intermittents ont été examinés. Vingt-et-un étaient atteints d'une maladie de Parkinson idiopathique, 17 d'une atrophie multi-systématisée, 1 d'un syndrome parkinsonien d'origine vasculaire, 1 d'un syndrome parkinsonien d'origine iatrogène et 2 d'un syndrome extrapyramidal non étiqueté. Tous avaient une rétention d'urine définie par un résidu postmictionnel supérieur ou égal à cent cinquante millilitres. Tous étaient symptomatiques et les symptômes rapportés étaient une dysurie (30/42), une hyperactivité vésicale (20/42) et une incontinence urinaire d'effort (6/42). La cystomanométrie mettait en évidence une hyperactivité détrusorienne (15/42), une dyssynergie vésico-sphinctérienne (15/42), une hypo-contractilité détrusorienne (14/42), une incompétence sphinctérienne (4/42) ou un défaut de compliance (3/42). Tous les patients maitrisaient la technique des auto-sondages intermittents. Quatorze (33\%) ont arrêté l'auto-sondage précocement dans les mois qui suivaient l'apprentissage en raison du retentissement fonctionnel de la dégradation neurologique.

Conclusion: Même si un tiers des patients abandonnent la réalisation des auto-sondages en raison de l'aggravation de la maladie neurologique, ces derniers restent la méthode de choix du traitement symptomatique de la rétention chronique du patient parkinsonien. Mots clés: "syndromes parkinsoniens ", " auto-sondage », " rétention urinaire » 


\title{
Self-intermittent-catheterization and urinary retentions in parkinsonian syndromes
}

\begin{abstract}
Introduction : Lower urinary tract symptoms are common in Parkinson diseases, especially chronic urinary retention. In case of significant and symptomatic post void residual, a specific treatment is necessary in order to empty the bladder and nowadays the gold standard of a such neurogenic bladder is based on self-intermittent-catheterizations if possible at all. We carried out a retrospective study about feasibility and outcomes of selfintermittent-catheterizations in this population.

Methods : Retrospective study with qualitative and quantitative, clinical and instrumental (urodynamic) assessment, of lower urinary tract symptoms as urinary retention in extrapyramidal syndromes.
\end{abstract}

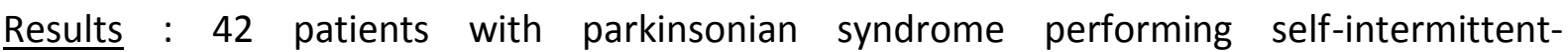
catheterization were assessed. Twenty-one had idiopathic Parkinson's Disease, 17 Multiple System Atrophy, 1 vascular Parkinson, 1 iatrogenic Parkinson and 2 not yet determined parkinsonian syndromes. All the patients had urinary retention characterized by a postvoiding residual volume more than $150 \mathrm{ml}$. All the patients were symptomatic and reported voiding dysfunction (30/42), overactive bladder syndrome (20/42), stress urinary incontinence (6/42). The cystometry showed detrusor overactivity (15/42), bladdersphincter dyssynergia (15/42), detrusor underactivity (14/42), sphincter deficiency (4/42) or bladder compliance alteration (3/42). Each patient have learned and well controlled selfintermittent-catheterization technique. Fourteen (33\%) stopped self-intermittentcatheterization prematurely in the following months because of functional impact of neurological worsening.

Conclusion : Even if one third of the patients had stopped self-intermittent-catheterization because of neurological deterioration, this technique remains the gold standard for the treatment of urinary chronic retention in parkinsonian patients.

Key words : Parkinson disease, urinary retention, self-intermittent-catheterization 
1

2

3

4

7

8

10

11

12

13

14

15

16

17

18

19

20

21

22

23

24

25

26

27

28

29

30

31

32

33

34

35

36

37

38

39

40

41

42

43

44

45

46

47

48

49

50

51

52

53

54

55

56

57

58

59

60

61

62

63

64

65 


\section{Introduction}

Les troubles mictionnels de la maladie de Parkinson et des syndromes apparentés, ont été largement rapportés $(1,2)$. A côté des anomalies de la phase de remplissage le plus souvent illustrées par un syndrome clinique d'hyperactivité vésicale, la dysurie et les rétentions chroniques d'urine sont fréquentes. La physiopathologie de ces dernières reste complexe et multimodale (hypoactivité détrusorienne, hyperactivité ou dyskinésie sphinctérienne) alors que l'étiopathogénie est souvent multifactorielle (neurogénique et obstructive) tout particulièrement chez l'homme en raison de la prévalence de l'hypertrophie bénigne prostatique dans la tranche d'âge où se rencontre la maladie de Parkinson. La typologie des troubles urinaires et plus spécifiquement l'existence d'une telle rétention, peut aussi constituer un marqueur de gravité voire entrer dans la discussion de l'étiologie même du syndrome parkinsonien quand on connaît la prévalence de la rétention urinaire dans les syndromes extrapyramidaux évoluant dans le cadre des AMS.

Au-delà de la caractérisation diagnostique, la rétention urinaire des syndromes extrapyramidaux pose des questions d'ordre thérapeutique. En effet au-delà de la discussion de leur efficacité pas toujours démontrée en cas de dysurie neurogénique, les alphabloquants posent toujours le problème de leur tolérance en raison de la possible majoration des manifestations d'hypotension orthostatique souvent observées dans le cadre des manifestations dysautonomiques des syndromes extrapyramidaux. De plus, les drogues parasympathomimétiques directes ou indirectes (anti-cholinestérasiques) ont démontré leur peu d'intérêt dans le traitement de la rétention et sont de plus souvent antinomiques par rapport à certaines médications souvent prescrites au cours des syndromes parkinsoniens (cholinestérasiques) qu'elles peuvent antagoniser. Reste alors le gold standard du traitement de la rétention urinaire neurologique que constituent les auto-sondages intermittents (1) (2). Cette thérapeutique, pourtant largement utilisée dans nombre de pathologies neurologiques (lésions médullaires traumatiques, sclérose en plaques, neuro-vessie diabétique, neuropathies périphériques, rétentions neurogènes post chirurgie du petit bassin), n'a jamais été évaluée au cours des syndromes extrapyramidaux. Si son intérêt potentiel est indéniable, les caractéristiques fonctionnelles des syndromes parkinsoniens (tremblements, bradykinésie, hypertonie, rigidité axiale) peuvent potentiellement perturber apprentissage, réalisation, adhérence et compliance à ce traitement. II nous a donc paru 
opportun de vérifier la faisabilité et l'adhérence aux auto-sondages dans une population extrapyramidale avec rétention urinaire symptomatique impactant le pronostic médical et la qualité de vie des patients.

\section{Méthodes}

II s'agit d'une étude rétrospective descriptive analysant les données qualitatives et quantitatives d'une population de patients avec syndrome parkinsonien ayant une rétention urinaire chronique et chez qui la technique des auto-sondages intermittents a été initiée. La recherche a été menée sur la base des données d'un service de neuro-urologie d'un hôpital universitaire. Tous les syndromes parkinsoniens étaient inclus via les mots clés suivants : "Parkinson ", " maladie de Parkinson idiopathique ", " atrophie multi-systématisée », " atrophie olivo-ponto-cérébelleuse ", " dégénérescence striato-nigrique », " syndrome de Shy-Dragger ", "paralysie supra nucléaire progressive» "maladie de SteeleRichardson ", " démence à corps de Lewy ", " syndrome parkinsonien », " dégénérescence cortico-basale ». La rétention d'urine était définie par un résidu post-mictionnel supérieur ou égal à cent cinquante millilitres. Le syndrome dysurie-rétention était toujours symptomatique (pollakiurie réactionnelle, fuites par regorgement, infections à répétitions notamment fébriles). Chaque dossier a été traité pour extraire les données cliniques (typologie des troubles vésico-sphinctériens, questionnaires de symptômes, données démographiques), les données urodynamiques (cystomanométrie, sphinctérométrie, électrophysiologie périnéale), les comorbidités et les traitements particulièrement des affections urologiques et gynécologiques associées; la typologie du syndrome extrapyramidal et son étiologie précise ; les caractéristiques des auto-sondages (fréquence, efficacité, complications, durée) et les traitements à visée urinaire associés.

\section{Résultats}

\section{Population et comorbidités}

Quarante-deux patients atteints de syndrome extrapyramidal ont été analysés. La population comprenait 18 femmes et 24 hommes d'âge moyen 68 ans. Les étiologies se répartissaient en 21 maladies de Parkinson idiopathique (50\%), 17 atrophies multisystématisées (57 \%), 1 syndrome parkinsonien d'origine vasculaire, 1 syndrome parkinsonien d'origine iatrogène post-neuroleptiques et 2 syndromes extrapyramidaux non encore étiquetés. Tous les patients avaient un traitement antiparkinsonien, dont deux par 
stimulation cérébrale profonde. La plupart des patients n'étaient pas en activité professionnelle (retraite, invalidité, arrêt de travail). Sur les 24 hommes, 13 (54\%) avaient une hypertrophie prostatique dont 7 opérées. Les 6 autres n'avaient pas de syndrome obstructif à l'étude pression-débit témoignant du caractère non symptomatique de l'adénome sur la phase mictionnelle et donc d'une dysurie strictement neurogénique. Parmi les comorbidités, 3 patients avaient une atteinte radiculaire associée (canal lombaire étroit, hernie discale), 4 patientes avaient des antécédents de chirurgie d'incontinence urinaire (bandelettes sous-urétrales non responsables de la rétention-dysurie), 3 avaient eu une cure de prolapsus génital. On retrouvait encore deux patients aux antécédents de méga-vessie congénitale (3) et un cas de maladie du col au stade de sclérose et opérée. Tous les patients étaient rétentionnistes et exprimaient dans à $71 \%$ une dysurie, $48 \%$ un syndrome clinique d'hyperactivité vésicale et dans $14 \%$ des cas une incontinence urinaire à l'effort ( 4 femmes et 2 hommes).

\section{Résultats urodynamiques}

Le bilan urodynamique retrouvait une hyperactivité détrusorienne pour $38 \%$ (16/42) des patients dont deux avaient un régime à haute pression endo-vésicale, une dyssynergie vésico-sphinctérienne pour $36 \%$ (15/42) des patients, une hypo-contractilité détrusorienne pour $33 \%$ (14/42) des patients, une incompétence sphinctérienne pour quatre patients, un défaut de compliance pour trois patients ( 2 maladies de Parkinson idiopathique, 1 atrophie multi-systématisée). Nous n'avons pas pu mettre en évidence de différences significatives concernant la typologie clinique et urodynamique entre syndromes extrapyramidaux atypiques et maladie de Parkinson idiopathique (MPI), probablement en raison de la sélection des patients ayant une MPI par le filtre de la rétention d'urine.

\section{Auto-sondages}

L'instauration des auto-sondages survenait en moyenne à cinq ans de l'évolution des symptômes parkinsoniens. Nous n'avons pas utilisé de scores spécifiques pour apprécier la gravité fonctionnelle du parkinsonisme (UPDRS, Hoehn \& Yahr ...) $(4,5)$. La survenue de la rétention urinaire dans la maladie de Parkinson idiopathique était plus tardive (6 ans) que dans les atrophies multi-systématisées ( 2 ans) de manière significative (t de Student -3,28; IC $95 \%[-6,77 ;-1,55], p=0,003)$. L'arrêt des auto-sondages survenait chez quatorze patients, sept atrophies multi-systématisées et six maladies de Parkinson idiopathiques (NS, P=0.41). 
Les raisons étaient la dégradation neurologique permanente ou transitoire, la difficulté au sondage, le temps passé à effectuer le sondage. Les difficultés rapportées étaient des problèmes d'équilibre notamment en raison de la cyphose, de la rigidité axiale et donc de tenue du tronc avec instabilité posturale, les difficultés à écarter les lèvres ou à repérer le méat urétral chez la femme, des difficultés de progression de la sonde, des douleurs. Sept patients, en raison d'une hyperactivité vésicale, prenaient un traitement parasympathycolytique, quatre prenait un traitement alpha-bloquant (tous des maladies de Parkinson idiopathique). Il y avait trois patients atteints d'atrophies multi-systématisées avec dysautonomie associée (avec notamment une hypotension orthostatique majeure). Tous les patients avaient un syndrome végétatif habituel (hypotension orthostatique). Concernant les questionnaires liés aux auto-sondages, tous les scores du questionnaire IC-DI-Q (6) étaient inférieurs à 10/78 témoignant de peu de difficultés rapportées lors des auto-sondages; tous les scores InCaSaq (7) étaient inférieurs à 9/24 sauf un (20/24) témoignant d'une bonne satisfaction : tous les scores I-CAS étaient inférieurs à $1 / 8$ sauf un $(6,75)$ témoignant d'une forte adhérence aux auto-sondages.

\section{Discussion}

\section{Rétention urinaire chronique}

Cette étude démontre la faisabilité, l'innocuité et l'efficacité des auto-sondages intermittents dans la rétention chronique des syndromes parkinsoniens. A notre connaissance, il s'agit de la première étude de ce type. La rétention urinaire fait partie des symptômes urinaires fréquemment observés au cours des syndromes extrapyramidaux (40 \%). La typologie urinaire est différente entre la maladie de Parkinson idiopathique et les atrophies multi-systématisées, constituant ainsi une des arguments diagnostiques discriminatifs entre ces formes cliniques (1) (8). C'est ainsi que la rétention urinaire est plus fréquente au cours des atrophies multi-systématisées que dans la maladie de Parkinson idiopathique. Cette rétention d'urine pose le problème de sa physiopathologie et de son étiopathogénie dans la mesure où les syndromes extrapyramidaux apparaissent dans une population âgée (9), où des comorbidités urologiques associées peuvent être elles-mêmes facteurs de rétention. Ce n'est habituellement pas le cas pour d'autres pathologiques neurologiques où la rétention est très prévalente comme la paraplégie traumatique ou la sclérose en plaques puisqu'il s'agit de pathologies de femmes et d'hommes jeunes. 
L'hypertrophie bénigne prostatique pose ainsi toujours le problème de son éventuelle responsabilité dans la rétention urinaire chronique (ou aigüe) de l'homme parkinsonien rendant difficile la stratégie thérapeutique. Le bilan urodynamique avec étude pressiondébit à la recherche d'un syndrome obstructif (augmentation des pressions permictionnelles couplées à la diminution du débit) et éventuellement la positivité d'un test à l'endo-prothèse endo-urétrale permettent en règle de préciser au mieux le facteur à incriminer (neurogénique, obstructif). Cependant, même dans le cas d'une responsabilité avérée d'une obstruction, il n'est pas toujours licite de proposer un geste chirurgical compte tenu d'une part de l'hypo-contractilité-hypokinésie détrusorienne extrapyramidale (10) associée qui persisterait en post-opératoire à la levée de l'obstacle; et d'autre part dans le cadre d'atrophies multi-systématisées par l'existence d'une dénervation des muscles périnéaux par lésion des noyaux d’Onulf responsable d'une incompétence sphinctérienne qui pourrait s'exprimer par des fuites à la levée de l'obstacle prostatique. Enfin, on ne dispose pas de thérapeutique médicale efficace et sécuritaire pour soit améliorer la contraction détrusorienne dans le cadre d'une hypo-contractilité neurogénique (parasympathycomimétiques directs ou indirects); soit diminuer la composante dyssynergique fonctionnelle urétrale en raison du risque des alpha-adrénergiques chez les patients aux troubles dysautonomiques. Les auto-sondages intermittents s'avèrent donc être la thérapeutique de choix pour gérer cette rétention dont le traitement est justifié d'une part par le risque inhérent à la rétention (infection urinaire, altération du haut appareil urinaire mais si elles sont plus rares que dans les lésions pyramidales), et d'autre part en raison des conséquences en terme symptomatique de la rétention incomplète à type de pollakiurie voir des fuites par regorgement. Il paraît dès lors pertinent de vérifier la faisabilité, l'efficacité, la persistance des auto-sondages dans une population de patients extrapyramidaux.

\section{Spécificités du parkinsonisme}

Notre étude démontre que les auto-sondages intermittents sont acceptés, bien tolérés et efficaces chez deux tiers des patients et peuvent donc être très raisonnablement proposés comme traitement à long terme. Néanmoins, dans un tiers des cas, ces auto-sondages génèrent des problèmes de compliance, d'adhérence et de persistance. Ceci est assez spécifique aux patients extrapyramidaux. En effet, les patients arrêtent en raison d'une dégradation neurologique progressive souvent inéluctable après la phase de « lune de miel » post induction de dopa thérapie, de difficultés au sondage (progression de la sonde, repère 
du méat), des problèmes d'équilibre, de temps long passé à effectuer le sondage. Ceci procède des déficits neuro-fonctionnels et neuro-orthopédiques induits par les syndromes extrapyramidaux tels que l'akinésie, l'hypertonie axiale et la rigidité, générant des difficultés positionnelles, mais aussi les troubles et instabilité de la posture induisant des troubles de l'équilibre et de la motilité. Le maintien de la gestuelle et des postures adaptées à l'autosondage est ainsi très problématique chez ces patients pouvant perturber la réalisation et la précision des gestes. La bradykinésie et l'hypométrie qui se traduisent par la lenteur et le défaut d'amplitude des mouvements, l'altération de la kinesthésie et de la coordination bimanuelle pénalisent le temps de préparation pour l'auto-sondage (toilette locale, préparation du matériel...) ainsi que sa réalisation (précision du sondage, progression de la sonde...). Parfois même, des troubles cognitifs parfois observés au cours des syndromes extrapyramidaux peuvent perturber le schéma moteur, l'apprentissage et le maintien de la réalisation des auto-sondages intermittents (11). De plus, on connaît la grande variabilité de l'état moteur des syndromes extrapyramidaux au cours de la journée avec parfois des phénomènes ON-OFF extrêmement perturbants lorsque I'horaire du sondage arrive lors d'une période OFF (12). L'éducation thérapeutique joue alors un grand rôle pour choisir avec le patient les moments pertinents en termes d'horaires d'auto-sondages, notamment par rapport au délai de prise de dopa-thérapie.

\section{Limitations}

En dépit de son caractère rétrospectif, cette étude semble intéressante du fait du nombre de patients réalisant les auto-sondages, l'importance de la technique dans la gestion de la rétention urinaire et les données cliniques et urodynamiques analysées. Une étude spécifique prospective permettrait de préciser la typologie des patients pouvant au mieux bénéficier et adhérer au traitement; et d'optimiser la qualité et la satisfaction des autosondages intermittents en termes de chronobiologie (prise de dopa-thérapie, aides ergothérapiques).

\section{Conclusion}

La pratique des auto-sondages intermittents est une technique efficace et sure au cours des syndromes parkinsoniens malgré un taux d'arrêt dans un tiers des cas. L'amélioration de la compliance et de l'adhérence peuvent procéder d'une adaptation du geste au cours de l'éducation thérapeutique avec une prise en compte de la spécificité de l'efficacité 
temporelle des médications antiparkinsonienne en modulant les séquences d'auto-sondages en fonction des prises de dopa-thérapie.

\section{RÉFÉRENCES}

1. Sakakibara R, Panicker J, Finazzi-Agro E, lacovelli V, Bruschini H, Parkinson's Disease Subcomittee, The Neurourology Promotion Committee in The International Continence Society. A guideline for the management of bladder dysfunction in Parkinson's disease and other gait disorders. Neurourol Urodyn. juin 2016;35(5):551-63.

2. Ogawa T, Sakakibara R, Kuno S, Ishizuka O, Kitta T, Yoshimura N. Prevalence and treatment of LUTS in patients with Parkinson disease or multiple system atrophy. Nat Rev Urol. févr 2017;14(2):79-89.

3. Falcou L, Mauruc E, Guinet-Lacoste A, Jousse M, Le Breton F, Vérollet $D$, et al. [Lazy bladder syndrome: review of 126 cases]. Progres En Urol J Assoc Francaise Urol Soc Francaise Urol. sept 2014;24(10):651-7.

4. Goetz CG, Tilley BC, Shaftman SR, Stebbins GT, Fahn S, Martinez-Martin P, et al. Movement Disorder Society-sponsored revision of the Unified Parkinson's Disease Rating Scale (MDS-UPDRS): scale presentation and clinimetric testing results. Mov Disord Off J Mov Disord Soc. 15 nov 2008;23(15):2129-70.

5. Goetz CG, Poewe W, Rascol O, Sampaio C, Stebbins GT, Counsell C, et al. Movement Disorder Society Task Force report on the Hoehn and Yahr staging scale: Status and recommendations The Movement Disorder Society Task Force on rating scales for Parkinson's disease. Mov Disord. 1 sept 2004;19(9):1020-8.

6. Guinet-Lacoste A, Jousse M, Tan E, Caillebot M, Le Breton F, Amarenco G. Intermittent catheterization difficulty questionnaire (ICDQ): A new tool for the evaluation of patient difficulties with clean intermittent self-catheterization. Neurourol Urodyn. janv 2016;35(1):85-9. 
7. Guinet-Lacoste $A$, Jousse $M$, Verollet $D$, Sheikh Ismael S, Le Breton $F$, Tan $E$, et al. Validation of the $\operatorname{InCaSaQ}$, a new tool for the evaluation of patient satisfaction with clean intermittent self-catheterization. Ann Phys Rehabil Med. avr 2014;57(3):159-68.

8. Gilman S, Wenning GK, Low PA, Brooks DJ, Mathias CJ, Trojanowski JQ, et al. Second consensus statement on the diagnosis of multiple system atrophy. Neurology. 26 août 2008;71(9):670-6.

9. Blin P, Dureau-Pournin C, Foubert-Samier A, Grolleau A, Corbillon E, Jové J, et al. Parkinson's disease incidence and prevalence assessment in France using the national healthcare insurance database. Eur J Neurol. mars 2015;22(3):464-71.

10. Terayama K, Sakakibara R, Ogawa A, Haruta H, Akiba T, Nagao T, et al. Weak detrusor contractility correlates with motor disorders in Parkinson's disease. Mov Disord Off J Mov Disord Soc. déc 2012;27(14):1775-80.

11. McDonald C, Winge K, Burn DJ. Lower urinary tract symptoms in Parkinson's disease: Prevalence, aetiology and management. Parkinsonism Relat Disord. févr 2017;35:8-16.

12. Dietrichs E, Odin P. Algorithms for the treatment of motor problems in Parkinson's disease. Acta Neurol Scand. 30 janv 2017; 
Tableau 1: Résultats des bilans urodynamiques chez quarante-deux patients extrapyramidaux en rétention urinaire chronique auto-sondés.

Table 1: Results of urodynamics findings for forty-two extrapyramidal patients in chronic urinary retention self-catheterized. 
Symptômes

Dysurie

Hyperactivité vésicale

Incontinence urinaire d'effort

Bilan urodynamique

Hyperactivité détrusorienne

Dyssynergie vésico-sphinctérienne

Hypo-contractilité détrusorienne

Incompétence sphinctérienne

Défaut de compliance détrusorienne
MPI

14

8

3

6

4

7

1

2
AMS

14

11

3

0

-

2

1
Autres

1

2

1

1

10

